\title{
Exploiting Trunked Radio to Support ITS Network Expansion and Redundancy
}

\author{
Ekasit Vorakitolan $^{\dagger}$, Joseph P. Havlicek ${ }^{\dagger}$, Mohammed Atiquzzaman ${ }^{\ddagger}$ and Ronald D. Barnes ${ }^{\dagger}$ \\ ${ }^{\dagger}$ School of Electrical and Computer Engineering $\quad{ }^{\ddagger}$ School of Computer Science \\ The University of Oklahoma \\ Norman, Oklahoma 73019 \\ Email: \{ekasitv, joebob, atiq, ron\}@ou.edu
}

\begin{abstract}
Typical intelligent transportation systems (ITS) are comprised of geographically distributed ITS devices including sensors, cameras and dynamic message signs (DMS). There are several options for providing data communication between these field devices and traffic management centers (TMC). Wireless networks are attractive due to their relatively lower cost and ease of deployment compared to fixed networks. However, these face unique security and signal interference problems, and deploying new wireless networks can require significant equipment investment.

In this work, a new extremely low-cost wireless strategy for ITS network communication is presented. This approach applies control channels of the Network Trunking System (NTS), a licensed radio frequency to support the voice communication, to control DMS operation under the National Transportation Communications for ITS Protocol (NTCIP). Because the NTS network has already installed in the metropolitan, urban, and rural areas in Oklahoma, it provides a cheap deployment solution requiring only additional adapter devices. Long term operation and maintenance costs can further be amortized between voice and data services. The proposed technique is compared to the existing ITS wireless networking strategies and hybrid network strategies merging wired and wireless networks deployed in Oklahoma. Details of the strategy for using wireless networking in the Oklahoma ITS and experiences with wireless ITS device deployment are also provided.
\end{abstract}

\section{INTRODUCTION}

This paper presents the use of the Oklahoma Department of Transportation (ODOT) licensed $150 \mathrm{MHz}$ frequency band NTS. This network has already been deployed across the state for voice communication, thus our approach avoids the expense of the deployment of a new dedicated network while still providing reliable communication with low-bandwidth ITS devices. This network can be used in conjunction with the wired, wireless, and hybrid networks that already support Oklahoma's ITS.

Communication networks play an important role in ITS, linking traffic monitoring and traveller feedback devices to TMCs. There are distinct tradeoffs in the choice of platform for these networks. Fiber-optic networks provide the highest throughput and data security, but are prohibitively expensive to be deployed outside densely populated areas. For example, the cost in 2009 to install a fiber optic network in San Francisco was between USD 95,000 and USD 240,000 per mile [1]. The use of telephone networks offers easy deployment (as long as the device is located in a service area). However, recurring service fees can become expensive for large numbers of widely deployed devices. They suffer from copper-wire line noise due to weather and humidity. Additionally, telephone service is not readily available at every roadside location.

Limitations of wired networks can constrain the deployment of ITS devices [2], [3]. For these reasons, wireless technologies including microwave and mobile telecommunications networks are frequently used as part of ITS networks. Public mobile telecommunications networks provide a quick and easy wireless solution, as service providers have already designed and installed networks that are typically shared with mobile phone users. Service providers are responsible for the network operation and maintenance. Use of these public wireless network also incurs monthly service fees, and they can suffer from a lack of security and limited coverage. The availability of these services is also not guaranteed during disasters and severe weather. Typical networks also provide greater data download bandwidth than upload, making it impossible to send the high resolution video streams back from fields to the TMC [3], [4].

Private wireless network offer many advantages for ITS applications. Both devices operating under licensed frequencies (e.g., ITS narrow band $220 \mathrm{MHz}$ and $4.9 \mathrm{GHz}$ band for public safety) and unlicensed frequencies (e.g., Industrial, Scientific and Medical (ISM) band $900 \mathrm{MHz}$ and $5.7 \mathrm{GHz}$ ) are utilized in the ITS applications [5]. These private wireless solutions provide more reliability, throughput, and security than public services. The use of licensed wireless device can reduce the problem of signal interference compared to unlicensed band because only authorized users can propagate that frequency into the air. The $220 \mathrm{MHz}$ band serves data transfer for traffic sensors, changeable message signs, and incident response vehicles [6]. However, seperate wireless solutions require significantly more network investment and maintenance than public telecommunication services. Additionally, although the $220 \mathrm{MHz}$ is used successfully in lowerdata-rate ITS applications, high efficiency wireless devices are required due to the limitation of bandwidth and problems such as fading, and multi-path signal propagation [7].

The unlicensed frequency devices used in the Oklahoma ITS are described in Section II. Building our experiences in wireless ITS deployments, our novel use of a $150 \mathrm{MHz}$ trunked wireless network is described in Section III. Results 


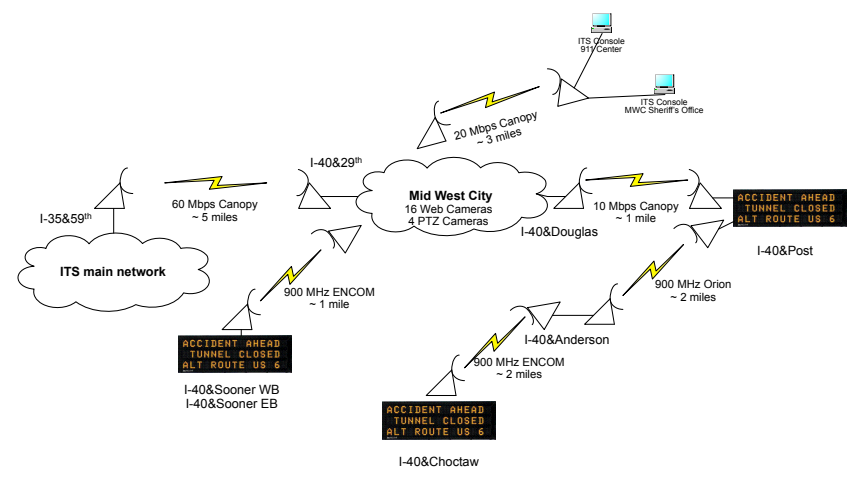

Fig. 1. The wireless network link between Oklahoma City main ITS network and Midwest City metropolitan network.

of our experimental testing of this approach are provided in Section IV followed by conclusions in Section V.

\section{Existing Wireless DeVices in The OKLAhoma ITS}

Like that of many other states, the Oklahoma ITS network is deployed with a fiber optic network backbone and utilizes wireless networks where fiber optic and other wired networks are unavailable. The specifications for the wireless equipment in use in the Oklahoma ITS are shown in Table I. ENCOM and Orion radio units are operated at lowest data throughput while the Motorola Canopy equipment provides the highest data throughput. Note that the $900 \mathrm{MHz}$ and $5.7 \mathrm{GHz}$ frequencies are unlicensed and open to public use. Since they can be easily used by the consumer devices, these frequencies are broadly used by individuals and are subject to interference.

In addition to interference and other noise issues, security is a significant concern when wireless networking is used for sensitive ITS functions. Data encryption is one method to address these security concerns. The encryption method used by each wireless device is also shown in Table I. The Federal Communications Commission (FCC) output power limit for each device and their operating temperature are other significant parameters to consider. Temperature constraints are of particular concern when wireless devices are selected for use in Oklahoma because its climate faces both harsh winter and summer seasons. Equipment must be able to operate in a wide range of temperature. Each of wireless units described in Table I have both advantages and disadvantages for particular ITS applications. The remainder of this section describes how each wireless devices are deployed in three different categories of ITS application within the Oklahoma ITS network.

\section{A. Small Data Network Applications}

The small data network category of applications is specified to support devices at the end of the network branch. These are typically low data transmission rate devices such as DMS or traffic sensors. In the Oklahoma ITS, a typical deployment in this category utilizes $900 \mathrm{MHz}$ radio devices. Figure 1, shows one such deployment. In this case, the DMSs in the Midwest City, OK area communicate with the Oklahoma ITS network using $900 \mathrm{MHz}$ radio. This radio link is operated in a point to multipoint configuration. This can be seen in this example with the radio link between the I-40\&29th Street location, the DMS at I-40\&Sooner Road (east bound), and I-40\&Sooner Road (west bound). For the DMSs at the I-40\&Post Road and I-40\&Choctaw Road, the point to point topologies of 900 $\mathrm{MHz}$ radio are used to carry the control data traffic. Because of the limitation of the line of sight between the radio links, a repeater is installed where obstructions occur between them.

For example, the repeater at the I-40\&Anderson Road is used to retransmit the signal between the signs on Choctaw Road and Post Road to overcome a large hill. In general, the $900 \mathrm{MHz}$ radio provides a low data transfer speed that is not suitable to carry traffic-camera video. However, for small spur network tasks, such as DMS control, the $900 \mathrm{MHz}$ radio is a suitable as communication medium because the DMS control commands are small data transfers. In fact, for permanent DMSs in Oklahoma, dial-up modems are used the main control; wireless networks are used for redundant back up. Existing wireless network devices providing communication backup for DMSs in the Oklahoma ITS consist of CDMA modems, Motorola $10 \mathrm{Mbps}$ Canopy and $900 \mathrm{MHz}$ radio devices. In some circumstances, wired and wireless devices applied in a hybrid network branch. For example, a permanent DMS on the I-44 Turnpike between Oklahoma City and Tulsa required constructing an initial telephone cable at a cost of USD 40,000 to connect the DMS to the telephone network at the tollbooth approximately 2 miles away. Instead, a dialup modem was installed at the tollbooth with an auto-answer mode configuration. This modem was in turn connected to a $900 \mathrm{MHz}$ radio. The radio pair linking the phone network at the tollbooth and the DMS is configured for point-to-point tunneling, bridging the data between the DMS and dial-up modem. The small data network application configuration has been extremely useful in the Oklahoma ITS network. It has allowed expansion with devices at the edge of the fiber optic network and telephone network service.

\section{B. Medium Data Network Applications}

For the medium data network category of applications, the Canopy wireless devices with throughput of 10 or $20 \mathrm{Mbps}$ are used. Figure 1 shows an example in this category. TMC consoles in Midwest City, OK are linked to the fiber-optic network using a $20 \mathrm{Mbps}$ Canopy. Users at both the 911 center and the sheriff's office can view and control the ITS cameras without network lag. In another case, a 10 Mbps Canopy is installed to link the main network and an individual user at the Federal Highway Administration (FHWA) in Oklahoma City, OK. Because the user is authorized to control both cameras and DMSs, the data bandwidth is configured for asymmetric 40/60 uplink/downlink throughput.

In addition to these medium data rate applications, there are other situations where $900 \mathrm{MHz}$ radio devices fail to provide adequate communication due to high noise environments with surrounding interference. In these locations, $10 \mathrm{Mbps}$ Canopy devices are used to connect DMSs to the ITS network in 
TABLE I

SUMMARY OF WIRELESS DEVICES USING IN THE STATE OF OKLAHOMA FOR ITS APPLICATIONS.

\begin{tabular}{|c|c|c|c|c|}
\hline & Orion [8] & ENCOM [9] & Motorola Canopy [10] & Motorola Canopy [11] \\
\hline Model & $900 \mathrm{MHz}$ OFDM & COMMPAK IP & $\mathrm{AP}, \mathrm{SM}$, or $\mathrm{BH}$ & 30/60 Mbps Backhaul \\
\hline Operating Frequency & $902-928 \mathrm{MHz}$ & 902-928 MHz & $\begin{array}{l}5.725 \mathrm{GHz} \text { to } 5.850 \mathrm{GHz} \\
4.940 \text { to } 4.990 \mathrm{GHz}\end{array}$ & $\begin{array}{l}5.47 \mathrm{GHz} \text { to } 5.725 \mathrm{GHz} \\
4.940 \text { to } 4.990 \mathrm{GHz}\end{array}$ \\
\hline Bandwidth & 5,10 , or $20 \mathrm{MHz}$ & N/A & $2.5 \mathrm{MHz}$ & $11 \mathrm{MHz}$ \\
\hline Transmission Method & Fixed Frequency & Frequency Hopping spread spectrum & Fixed Frequency & Fixed Frequency \\
\hline Modulation & OFDM, BPSK, or QPSK & N/A & FSK & BPSK,QPSK,16/64 QAM \\
\hline Data Speed & $5.5,11$, or $22 \mathrm{mbps}$ & $300 \mathrm{bps}$ to $230.4 \mathrm{kbps}$ & $10 \mathrm{mbps}$ or $20 \mathrm{mpbs}$ & $30 \mathrm{Mbps}$ or $60 \mathrm{Mbps}$ \\
\hline Interface & Ethernet(RJ-45) & $\begin{array}{l}\text { Ethernet(RJ-45), RS-232, } \\
\text { RS-422/485 (DB-9) }\end{array}$ & Ethernet(RJ-45) & Ethernet(RJ-45) \\
\hline Data Encryption & Dynamic key & $\begin{array}{l}128 \text { bit WEP, } 128 \text { bit WPA, } \\
256 \text { bit AES }\end{array}$ & AES & AES \\
\hline Error Detection & N/A & 32 bit CRC, ARQ & FEC and ARQ & FEC and ARQ \\
\hline Transmission power & up to $+30 \mathrm{dBm}$ & $+20 \mathrm{dBm}$ to $+30 \mathrm{dBm}$ & $+23 \mathrm{dBm}$ to $+30 \mathrm{dBm}$ & $+19 \mathrm{dBm}$ to $+25 \mathrm{dBm}$ \\
\hline Operating Temperature & -20 to +55 degree $\mathrm{C}$ & -40 to +75 degree $\mathrm{C}$ & -40 to +55 degree $\mathrm{C}$ & -40 to +60 degree $C$ \\
\hline
\end{tabular}

the same manner as in the small data network applications. Because the Canopy provides only an Ethernet interface, an Ethernet to serial converter is used to adapt the Canopy for communication with the DMS. In one deployment, a DMS in Tulsa, OK is located roughly 3 miles away from a division office. The telephone network does not reach a point close to the DMS. As in the application described in Section II-A, an auto-answer dial-up modem is installed at the division office. A pair of Ethernet-to-serial converters are used with point to point tunneling. Using the first, a the regular dial-up modem's RS-232 serial signal is converted to an Internet Protocol (IP) signal that is connected to the Canopy. At the DMS site, the IP signal received from the Canopy is connected through the second Ethernet-to-serial converter to change the IP signal back into an RS-232 signal that is sent to the sign controller.

\section{Large Data Network Applications}

Canopy $60 \mathrm{Mbps}$ equipment is used to bridge metropolitan or other urban area to the main backbone network in the large data network application. As an example, the ITS devices in the Midwest City, OK area consisting of 4 analog cameras, 16 digital web cameras, 3 permanent DMSs, and TMC consoles as shown in Figure 1. A Canopy 60 Mbps wireless link is used to bridge the data communication between this spur network and the ITS backbone network. In this case, the Canopy's data throughput is configured as symmetric (50/50 uplink/downlink) since video streams are transmitted back and forth between the main ITS network and the spur based on user request. This allows TMC console users in Oklahoma City and other ares on the network to control and view camera video in the Midwest City area and vice versa.

Wireless ITS deployments in Oklahoma have typically depended on public mobile network services and licensefree frequency devices. While private wireless networking using $900 \mathrm{MHz}$ radio or Canopy links are the most reliable redundant network connection for DMS devices, these approaches unfortunately can only extend to limited areas. Since the fiber optic network does not reach the majority of rural areas in the state, public network services are the only available form of secondary communication between rural DMSs and TMC consoles. This is a significant issue as these DMSs are increasingly being used to improve public safety through communication to travelers. In the next section, we provide a novel wireless communication strategy that is used to link otherwise wirelessly unreachable DMSs using an existing private voice communication network.

\section{DMS CONTROL USING AN EXISTING NeTWORK TRUnKED RAdio SyStem}

ODOT's $150 \mathrm{MHz}$ frequency-band NTS network has been deployed statewide, covering metropolitan, urban, and rural areas with 96 base stations around the state. Each base station utilizes a dipole omni-directional antenna. Coverage varies by station with a maximum range of approximately 20 miles. The state is divided into eight geographical regions [12] and frequency channels are assigned to each region. The backbone network for NTS for each region is connected together using a wired network such as fiber optic or T-1 leased line services. The NTS is based on the Passport protocol, a standard for the Logic Trunked Radio (LTR) developed by Trident Micro Systems [13]. Unlike other states that have invested in dedicated, private ITS radio networks such as $220 \mathrm{MHz}$ systems, we have utilized the NTS gateway software, TrAVL Bridge, to connect a Skyline DMS server's Rs-232 serial interface to the NTS network. Trident RTU units with quarter-wave dipole antennae are installed at each sign to translate the control Rs232 serial data back to the sign's controller.

Both data and voice are sent using frequency modulation (FM) on the NTS system. For communicating with DMS, we use the data channel assigned for messaging control. This messaging control channel uses FM $12.5 \mathrm{KHz}$ narrowband 


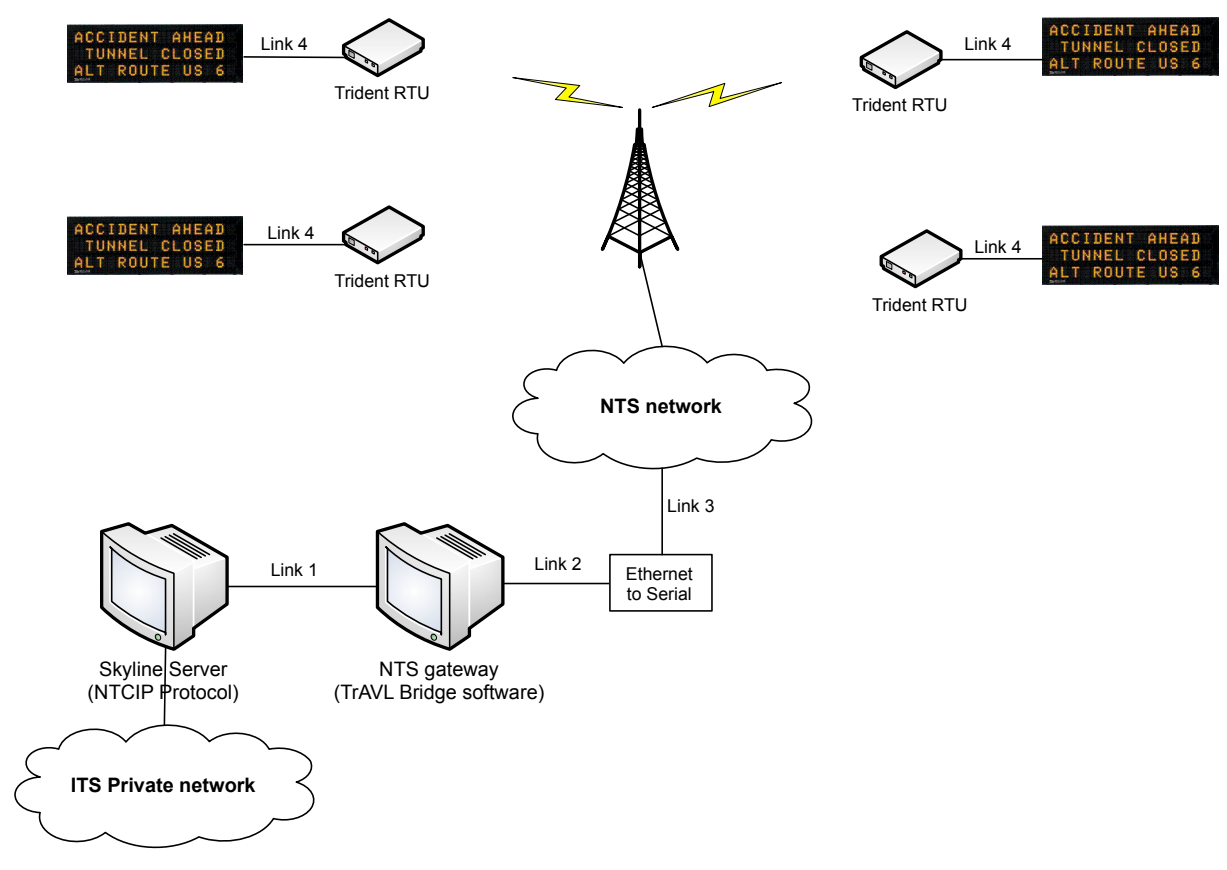

Fig. 2. Network interface through NTS.

spacing $^{1}$. The channel is calibrated with a $1000 \mathrm{MHz}$ tone for less than a $800 \mathrm{~Hz}$ deviation. With this bandwidth allocation, 9600 bps data transfer should be supported. However, to ensure reliable data communication between the DMS and the center a 1200 bps data rate is used for DMS control.

The network diagram of the DMS server software interface to the NTS is shown in Figure 2. The NTS network is used to provide communication between the Skyline software and the DMS in the field using this existing wide-area radio network. Link 1 is a network link between the Skyline server and the NTS gateway. The link establishes IP communication of eXtensible Markup Language (XML) data. Link 2 is a connection between the NTS gateway and the ethernet-toserial converter. The signal output from the ethernet-to serial converter is sent to the NTS network by Link 3. Using this link, control data is sent to all main network areas using the available NTS network control channel to transmit the sign controller commands to DMSs. At the sign, an RTU unit coverts the data back to RS-232 serial data which controls signs via $\operatorname{Link}(\mathrm{s}) 4$.

The basic idea of this approach is to construct a system that allows the DMS server and distant clients to operate as though they were directly connected (using a virtual communication port), utilizing a point-to-multipoint network. Regardless of direction, data is presented to the NTS Network in its native form (NTCIP protocol), conditioned for transport by the NTS Network, and finally delivered by the NTS Network to the target after being restored to its native NTCIP data package. The NTS gateway identifies sign addresses in the message from the Skyline software, and performs a database lookup to

\footnotetext{
${ }^{1}$ FCC Emission Designator 11K0F9W (narrowband data)
}

associate the target sign with a radio. The exchange is then initiated with the correct sign.

\section{Testing Results of Utilizing the NTS Network}

A testing network was setup at the ODOT central office by installing the NTS gateway and connecting it to the DMS server controller. The controller did not require any special setup since the NTS gateway software was configured to utilize a virtual serial port. Three RTU units are installed at DMSs in Oklahoma City: I-40 and Choctaw Road (location 1), I35 and 19th Street (location 2), and I-35 and Wilshire Blvd. (location 3). Each location is equipped with a different DMS model. Original communication with the DMS at location 1 was through dial-up modem and a $900 \mathrm{MHz}$ radio with a 9600 bps data rate. Locations 2 and 3 have dial-up modems and CDMA modems as their communication methods. Note that while communication through a dial-up modem is expected to be slower than each sign's secondary, wireless method of communication, the wired method is chosen as the primary way of communicating with each DMS because it doesn't require transportation agents to be connected to the Oklahoma ITS network to control a sign.

Testing was performed at each location to measure the average latency for each communication method to accomplish various tasks. Four different types of commands were sent to each DMS. First, the sign status was polled checking that the sign is operational. Polling the sign checks the status of communication, main power, sign temperature, ambient temperature, sign controller, LED power supplies, brightness, control mode, and door alarm. If the sign has no malfunction, the message "ODOT TEST MESSAGE" was sent to the sign, 


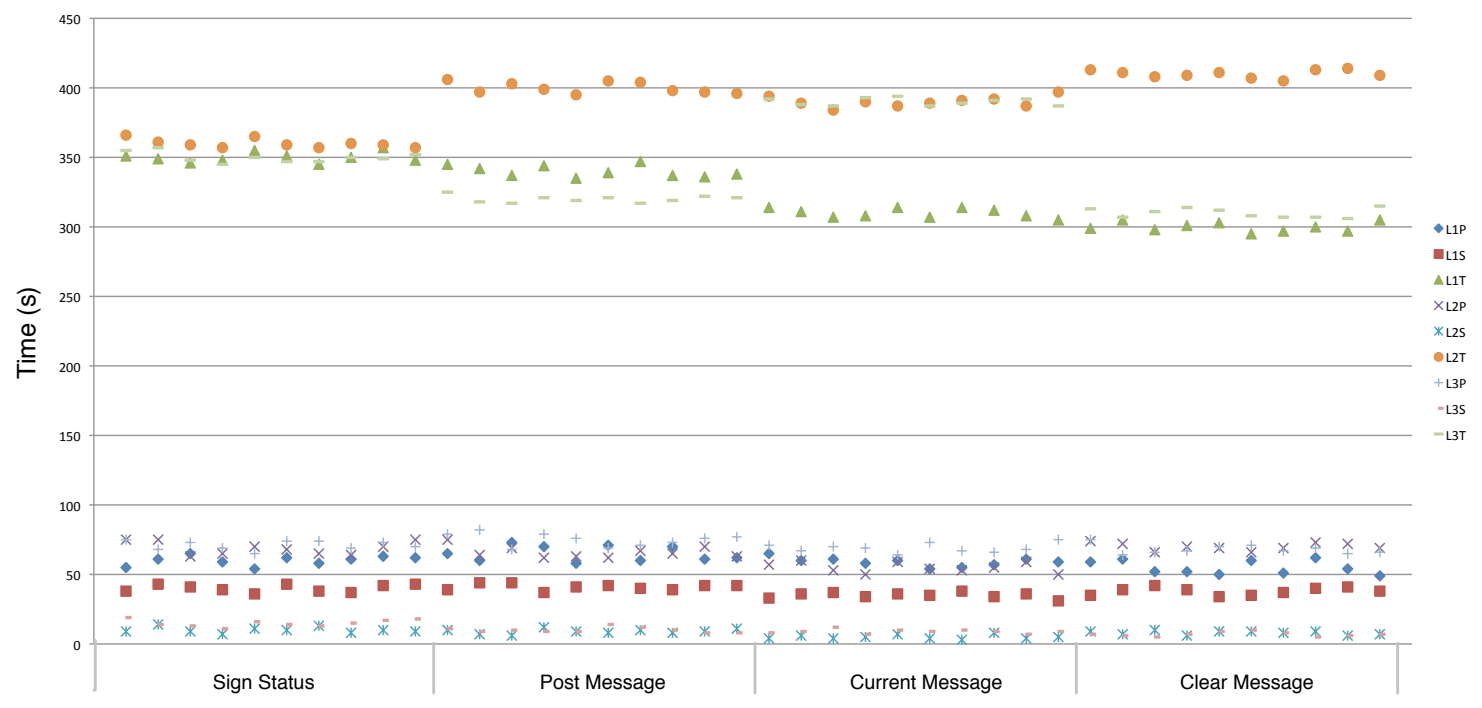

Fig. 3. Latency of sending various commands to message signs.

followed by a command to check the current message to verify that the new message was correctly posted to the sign. As last step, a final command was sent to clear the sign before repeating the test. The communication time for the transmission of each DMS command is shown in Figure 3. Results are shown for each of the three locations (L1, L2 and L3) using the primary $(\mathrm{P})$, secondary $(\mathrm{S})$, and the NTS network tested $(\mathrm{T})$ methods of communication. As expected, the secondary communication mechanism was always fastest followed by the primary dial-up communication. While sending commands through the NTS network was the slowest method of communication in each test, all tests completed in less than 7 minutes. While this latency may be unacceptable for many ITS functions, it is typically acceptable for the infrequent control of a DMS.

Note that although the dial up modem operates at $9600 \mathrm{bps}$ ( 8 times faster than the 1200 bps rate of the tested approach), it spends some time waiting in the initial process of original connection. Telephone line noise reduces the achieved data rate and can make the initial process unsuccessful. When an initial call fails, the server will automatically call back. In our tests, connections were always made after no more then two tries. This make the average time for the dial up modem up to about one minute for each category of command sent to the sign. Unlike dial up modem, the ENCOM $(900 \mathrm{MHz})$ radio is operated at 9600 bps does not requires an initial process to originate call. Hence, the communication time interval for sending message to signs is shorter than utilizing the dialup modem. The NTS network can provide maximum speed at $1200 \mathrm{bps}$ and needs at least 5 to 7 minutes for each transaction to complete. It does not require a initial process to originate call but it does require a process for NTS gateway to search the database for the sign address (multi-drop address) and find an available control channel to send to data to appropriate locations. To achieve stronger signal levels, we also found during our experiments that the RTU unit antenna needed to be installed outside of the sign. When the signal strength is weak, this directly effects data transition speed causing communication delays and timeouts.

Little latency variation is seen between test performing each of the various DMS commands. However, as the slowest method of communication, sending the message though the NTS network was slightly more dependent on the type (and the length) of the command being sent. As shown in Figure 3, each test was repeated 10 times for each sign. The results of these experiments are shown in Figure 3 and the average latency is reported in Table II along with the standard deviation for each set of tests and the maximum latency for that set. While the standard deviation in the latency of communication through the NTS network is higher than the secondary mode, less variation is actually seen in the NTS tests than in utilizing the dial-up modem.

The results from our experiments demonstrate that DMS control using the NTS network is possible. This approach provides alternative method of wireless communication to support ITS applications. Although the data throughput of this approach is not high as another wireless networking methods, it allows low-cost deployment of ITS devices at any region statewide. Because the NTS network is privately operated for the state's transportation agency, there is greater confidence of network availability during the severe weather or disaster relative to wireless services used by the public. With the cost shared for operation and maintenance with the existing voice communication application, the NTS further makes ITS network extension feasible within a limited budget.

\section{Conclusions}

Wireless networks are an attractive alternative to wired networks. They can bridge wired networks in several different network topologies such as those we have described as small, 
TABLE II

SUMMARY OF AVERAGE TIME, STANDARD DEVIATION, AND MAXIMUM TIME FOR COMMUNICATION TO DIFFERENT SIGN LOCATIONS THROUGH DIFFERENT NETWORK TYPES (TIMES IN SECONDS)

\begin{tabular}{|c|c|c|c|c|c|c|c|c|c|}
\hline & $\begin{array}{l}\text { Location } 1 \\
\text { Primary }\end{array}$ & $\begin{array}{l}\text { Location } 1 \\
\text { Secondary }\end{array}$ & $\begin{array}{l}\text { Location } 1 \\
\text { Testing }\end{array}$ & $\begin{array}{l}\text { Location } 2 \\
\text { Primary }\end{array}$ & $\begin{array}{l}\text { Location } 2 \\
\text { Secondary }\end{array}$ & $\begin{array}{l}\text { Location } 2 \\
\text { Testing }\end{array}$ & $\begin{array}{l}\text { Location } 3 \\
\text { Primary }\end{array}$ & $\begin{array}{l}\text { Location } 3 \\
\text { Secondary }\end{array}$ & $\begin{array}{l}\text { Location } 3 \\
\text { Testing }\end{array}$ \\
\hline Communication & Dialup & ENCOM & NTS & Dialup & CDMA & NTS & Dialup & CDMA & NTS \\
\hline Data rate(bps) & 9600 & 9600 & 1200 & 9600 & UDP & 1200 & 9600 & UDP & 1200 \\
\hline Sign status(Avg.) & 60 & 40 & 350 & 69 & 10 & 360 & 71 & 15 & 350 \\
\hline Sign status(Max.) & 65 & 43 & 357 & 75 & 14 & 366 & 75 & 19 & 357 \\
\hline Post message(Avg.) & 65 & 41 & 340 & 66 & 9 & 400 & 75 & 10 & 320 \\
\hline Post message(SD) & 5.23 & 2.14 & 3.97 & 4.02 & 1.73 & 3.87 & 4.38 & 1.79 & 2.37 \\
\hline Current message(SD) & 3.03 & 1.95 & 3.22 & 3.46 & 1.48 & 3.55 & 3.16 & 1.41 & 2.57 \\
\hline Current message(Max.) & 65 & 38 & 314 & 60 & 8 & 397 & 75 & 12 & 394 \\
\hline Clear message(Avg.) & 55 & 38 & 300 & 70 & 8 & 410 & 68 & 7 & 310 \\
\hline Clear message(SD) & 4.71 & 2.57 & 3.29 & 2.61 & 1.34 & 2.76 & 3.13 & 1.55 & 3.19 \\
\hline Clear message(Max.) & 62 & 42 & 305 & 74 & 101 & 414 & 75 & 10 & 315 \\
\hline
\end{tabular}

medium and larger data network applications. In order to reduce the investment cost, and improve data reliability and security, we have provided a new way use of an existing NTS radio to provide communication to DMSs. The results from our tests show that we can use the NTS as network medium to control NTCIP-protocol-based signs. Due to the small size of control messages, this data can be sent to through the NTS network using available control channels without interrupting the primary voice service. However, this DMS control is slow due to the maximum data rate at $1200 \mathrm{bps}$. Communication using our presented approach takes longer than that through telephone and CDMA modems where the data speed is 9600 bps. While this may appear slow compared to other networks but it is an extremely inexpensive way to implement ITS devices statewide including in rural regions. Sharing the NTS network for voice and data traffic also divides the cost of operation and maintenance. Other suitable low data rate ITS applications to utilize the NTS network include traffic sensors and portable DMSs Both need only to transfer a few bytes of data to TMC consoles. These traffic sensors and portable DMSs also frequently deployed in work zone areas. Data from devices monitoring traffic in these temporary locations can be easily communicated to the TMC via the NTS network. In the future, if the demand of the data is more than the control channel can support, it would also be possible to dedicate a voice channel for data use.

\section{ACKNOWLEDGMENT}

The authors would like to thank Alan Stevenson, ODOT ITS and Fiber Optic Engineering Manager, for his continued support of the OU ITS Lab. We would also like to thank Ty Todd, communication system manager at ODOT, who generously provided technical information regarding the NTS network. The authors would like express their appreciation to Dave Anderson, President/CEO at Trident Micro Systems, who permitted us to disclose the experimental results of communication with DMSs through the NTS network and provided testing equipment.

\section{REFERENCES}

[1] Columbia Telecommunications Corporation, "Brief engineering assessment: Efficiencies available through simultaneous construction and colocation of communication conduit and fiber," The National Association of Telecommunications Officers and Advisors and The City and Country of San Francisco, Tech. Rep., August 2009.

[2] Intelligent transportation systems (its) operations. Visited April, 2011. [Online]. Available: http://www.wsdot.wa.gov/Operations/ITS/

[3] A. Amanna, "Assessment of current and emerging broadband wireless technologies for VDOT's operations program," Virginia Tech Transportation Institute, Tech. Rep., Jun 2008.

[4] B. Kilani, E. Vorakitolan, J. Havlicek, M. Tull, and A. Stevenson, "Distributed ITS control and the oklahoma virtual TMC," in Proceedings of the 12th International IEEE Conference on Intelligent Transportation Systems, Missouri, USA, Oct. 2009, pp. 785-790.

[5] FCC rules for unlicensed wireless equipment operating in the ISM bands. Visited April, 2011. [Online]. Available: http://www.afar.net/ tutorials/fcc-rules/

[6] M. Fitz, J. Krogmeier, J. Grimm, J. gansman, T. Chen, and T. Magnusen, "The $220 \mathrm{MHz}$ ITS spectral allocation: Potential, pitfalls, and applications," IEEE Communicaitons Magazine, pp. 42-54, 1996.

[7] J. Grimm, M. Fitz, J. Krogmeier, T. Chen, T.Magnuse, J. Gansman, and W. Kuo, "High efficiency narrowband wireless modems for ITS applications," ITS Journal, vol. 3, no. 4, pp. 333-352, 1997.

[8] Orion $900 \mathrm{MHz}$ OFDM user manual. Visited April, 2011. [Online]. Available: http://www.wirelessinteractive.com/pdf/radios/ Orion900_manual.pdf

[9] ENCOM COMMPAK IP $900 \mathrm{MHz}$ and $2.4 \mathrm{GHz}$ serial/ethernet bridge. Visited April, 2011. [Online]. Available: http://www.encomwireless.com/document-centre/doc_view/ 142-commpak-ip?tmpl=component\&format=raw

[10] Canopy backhaul module user manual. Visited April, 2011. [Online]. Available: http://www.canopy-wireless-solutions.com/AppicationNotes/ BHManualIss5.pdf

[11] $4.9 \mathrm{GHz}$ wireless ethernet bridges. Visited April, 2011. [Online]. Available: http://www.canopy-wireless-solutions.com/AppicationNotes/ MotorolaPTP49400BridgeTechnicalSpecifications.pdf

[12] Oklahoma departemnt of transporation radio reference. Visited April, 2011. [Online]. Available: http://www.radioreference.com/apps/db/?sid= 5253

[13] PassPort trunking. Visited April, 2011. [Online]. Available: http: //www.tridentms.com/Home/PassPortTrunking/tabid/61/Defaultaspx 